„So schießt wieder einmal jeder auf jeden. Und natürlich sind immer die anderen schuld, dass der sehr kranke Patient Gesundheitssystem weiter darbt."

\title{
Schmerzspitzen
}

\section{Die Jagd ist auf!}

$\mathrm{J}$ edes Jahr zum Herbst ist die Jagd auf. So auch dieses Jahr in Wald und Flur und im Gesundheitswesen, allerdings mit ein paar anderen Vorzeichen als in den vergangenen Jahren. So wurde das sogenannte Antikorruptionsgesetz wegen Bestechlichkeit im Gesundheitswesen ( $\$ 299$ a und b) im Juni scharfgeschaltet und man höre und staune: In einem Jahr finden auch schon wieder die nächsten Bundestagswahlen statt.

Ein Jahr vor besagter Wahl werden Schuldige auch für die vom GKV-Spitzenverband angekündigte Erhöhung des Zusatzbeitrags gesucht. Und natürlich sind es wieder einmal die üblichen Verdächtigen, wie die AOK Bayern, deren Wahlspruch „Alles Ohne Kompromiss“ zu sein scheint, im August auf einer Pressekonferenz in München ausführte. Als Übeltäter ausgemacht wurden: korrupte, betrügerische Ärzte, Pflegeeinrichtungen, Dienstleister im Gesundheitswesen wie Sanitätshäuser und natürlich die Apotheker, dargestellt an entsprechenden Beispielen.

Aber auch die Krankenkassen, die sich gerne als Kreuzritter gegen den Missbrauch und die Korruption im Gesundheitswesen sehen, haben kein solch rein weißes Gewand, wie es die Kreuzritter seinerzeit trugen und die Krankenkassen heute für sich in Anspruch nehmen und zu vermitteln suchen.

Denn auch Krankenkassen zeigen Fehlverhalten im Gesundheitswesen, das aber in ihrem Fall auf einem anderen Gebiet liegt und von einer anderen Selbstkontrollinstitution aufgezeigt und geahndet wird. Zuständig für das hier angesprochene Fehlverhalten ist die Wettbewerbszentrale im hessischen Bad Homburg, denn im härter werdenden Wettbewerb der Krankenkassen werden auch die Sitten rauer. Werfen die Krankenkassen den Ärzten und anderen Leistungserbringern Fehlverhalten gegenüber den Kostenträgern vor, so zeigt die Wettbewerbszentrale Fehlverhalten der Kranken- kassen gegenüber ihren Kunden (Beitragszahlern) und Mitbewerbern auf.

Einer der Hintergründe für die raueren Sitten ist, dass der Spitzenverband der Krankenkassen angekündigt hat, dass viele gesetzliche Kassen den $\mathrm{Zu}$ satzbeitrag anheben werden. Im Jahr 2019 könnte er laut Doris Pfeiffer, Chefin des GKV-Spitzenverbands, bei über zwei Prozent liegen. Die für den Gesundheitsbereich zuständige Geschäftsführerin der Wettbewerbszentrale Cristiane Köber kann sich vorstellen, dass Tricksereien der Krankenkassen dann noch einmal zunehmen werden, da die eine oder andere Kasse sich etwas einfallen lassen müsse.

Aber auch der GKV-Spitzenverband steht in der Kritik und wurde von der Gesundheitsministerin des „Rebellen(frei)staats“ im Süden der Bundesrepublik - in dem der Verfasser dieses Beitrags übrigens gerne arbeitet -Melanie Huml (CSU) als realitätsfern, verspätet und zentralistisch kritisiert; der Beifall der bayerischen Ärzteschaft sicher dürfte ihr gewiss sein. Deshalb fordert sie Spitzenverbände auf Landesebene.

So schießt wieder einmal jeder auf jeden und natürlich sind die anderen schuld, dass der sehr kranke Patient Gesundheitssystem weiter darbt. Man fragt sich, ob es überhaupt noch um die Personen geht oder jemals ging, welche die einen Kunden und die anderen Patienten nennen. So gesehen wird mein Herzschmerz für die Sache Medizin wieder einmal auf die Spitze getrieben.

Mit der immer währenden Hoffnung auf Besserung verbleibt bis zum nächsten Mal

Ihr

Jörn Ludwig 